\title{
Canonical formulas via locally finite reducts and generalized dualities
}

\author{
Nick Bezhanishvili \\ Department of Philosophy \\ Utrecht University \\ Janskerkhof 13a, 3512 BL Utrecht \\ The Netherlands \\ N.Bezhanishvili@uu.nl
}

Axiomatizability, the finite model property (FMP), and decidability are some of the most frequently studied properties of non-classical logics. One of the first general methods of axiomatizing large classes of superintuitionistic logics (si-logics for short) was developed by Jankov 8. For each finite subdirectly irreducible Heyting algebra $A$, Jankov designed a formula that encodes the structure of $A$. The main property of the Jankov formula $\chi(A)$ is that a Heyting algebra $B$ refutes $\chi(A)$ iff $A$ is isomorphic to a subalgebra of a homomorphic image of $B$. In [9] Jankov utilized this method to show that there are continuum many si-logics; in fact, continuum many si-logics axiomatized by Jankov formulas. However, not every si-logic is axiomatizable by Jankov formulas.

Model-theoretic analogues of Jankov formulas were developed by de Jongh [10] for si-logics and by Fine [6] for modal logics. In [7] Fine introduced the concept of a subframe logic, axiomatized all transitive subframe logics by means of subframe formulas, and proved that each transitive subframe logic has the FMP. Zakharyaschev generalized Fine's approach, developed the model-theoretic theory of canonical formulas (in [12 for si-logics and in [11, 13] for modal logics), and showed that each si-logic and each transitive modal logic is axiomatizable by canonical formulas. See [5, Ch. 9] for an overview of these results.

In this talk, which is based on joint work with G. Bezhanishvili [1, 2, 3, 4, I will discuss an algebraic approach to the method of canonical formulas. I will mostly concentrate on the case of si-logics. But I will also review the case of modal logics and possible generalizations to substructural logics.

For si-logics the method boils down to identifying appropriate locally finite reducts of Heyting algebras. The variety of Heyting algebras has two well-behaved locally finite reducts, the variety of bounded distributive lattices and the variety of implicative semilattices. The variety of bounded distributive lattices is generated by the $\rightarrow$-free reducts of Heyting algebras, while the variety of implicative semilattices by the $\vee$-free reducts. Each of these reducts gives rise to canonical formulas that generalize Jankov formulas and provide an axiomatization of all si-logics.

For a finite subdirectly irreducible Heyting algebra $A$ and $D \subseteq A^{2}$, we design the $(\wedge, \rightarrow)$ canonical formula of $A$ that encodes fully the structure of the $\vee$-free reduct of $A$, and only partially the behavior of $\vee$. We also design the $(\wedge, \vee)$-canonical formula of $A$ that encodes fully the structure of the $\rightarrow$-free reduct of $A$, and only partially the behavior of $\rightarrow$. We prove that every si-logic is axiomatizable by $(\wedge, \rightarrow)$-canonical formulas as well as by $(\wedge, \vee)$-canonical formulas. We discuss the similarities and differences between these two kinds of formulas. Via the generalized Esakia duality of Heyting algebras and $(\wedge, \rightarrow)$-homomorphisms, we show that $(\wedge, \rightarrow)$-canonical formulas are algebraic analogues of Zakharyaschev's canonical formulas.

One of the main ingredients of our formulas is a designated subset $D$ of pairs of elements of a finite subdirectly irreducible Heyting algebra $A$. The obvious two extreme cases are when 
$D=\emptyset$ or $D=A^{2}$. When $D=A^{2}$, we show that the $(\wedge, \rightarrow)$ and $(\wedge, \vee)$-canonical formulas of $A$ are equivalent to the Jankov formula of $A$. On the other hand, when $D=\emptyset$, the $(\wedge, \rightarrow)$ canonical formulas produce the algebraic counterpart of subframe formulas, which axiomatize all subframe si-logics. In the $(\wedge, \vee)$-case, $D=\emptyset$ produces a new class of si-logics, which we term stable si-logics. As in the case of subframe logics, we prove that all stable si-logics have the FMP. We show that there are continuum many stable si-logics, and give examples showing that the classes of stable, subframe, and join-splitting si-logics are incomparable.

\section{References}

[1] G. Bezhanishvili and N. Bezhanishvili. An algebraic approach to canonical formulas: Intuitionistic case. Rev. Symb. Log., 2(3):517-549, 2009.

[2] G. Bezhanishvili and N. Bezhanishvili. An algebraic approach to canonical formulas: Modal case. Studia Logica, 99(1-3):93-125, 2011.

[3] G. Bezhanishvili and N. Bezhanishvili. Canonical formulas for wK4. Rev. Symb. Log., 5(4):731-762, 2012.

[4] G. Bezhanishvili and N. Bezhanishvili. Locally finite reducts of Heyting algebras and canonical formulas. 2013. Submitted.

[5] A. Chagrov and M. Zakharyaschev. Modal logic, volume 35 of Oxford Logic Guides. The Clarendon Press, New York, 1997.

[6] K. Fine. An ascending chain of S4 logics. Theoria, 40(2):110-116, 1974.

[7] K. Fine. Logics containing K4. II. J. Symbolic Logic, 50(3):619-651, 1985

[8] V. Jankov. On the relation between deducibility in intuitionistic propositional calculus and finite implicative structures. Dokl. Akad. Nauk SSSR, 151:1293-1294, 1963. (Russian).

[9] V. Jankov. The construction of a sequence of strongly independent superintuitionistic propositional calculi. Soviet Math. Dokl., 9:806-807, 1968.

[10] D. de Jongh. Investigations on the Intuitionistic Propositional Calculus. PhD thesis, University of Wisconsin, 1968.

[11] M. Zakharyaschev. Syntax and semantics of modal logics that contain S4. Algebra and Logic, 27(6):408-428, 1988.

[12] M. Zakharyaschev. Syntax and semantics of superintuitionistic logics. Algebra and Logic, 28(4):262-282, 1989.

[13] M. Zakharyaschev. Canonical formulas for K4. I. Basic results. J. Symbolic Logic, 57(4):1377-1402, 1992. 